Article

\title{
Propagation of Non-Linear Lamb Waves in Adhesive Joint with Micro-Cracks Distributing Randomly
}

\author{
Xiaoyang Li ${ }^{1}$, Guoshuang Shui ${ }^{1, *}$, Youxuan Zhao ${ }^{2,3}$ and Yue-Sheng Wang ${ }^{1,4, *(D)}$ \\ 1 Department of Mechanics, Beijing Jiaotong University, Beijing 100044, China; 15115313@bjtu.edu.cn \\ 2 College of Aerospace Engineering, Chongqing University, Chongqing 400044, China; \\ youxuan.zhao@cqu.edu.cn \\ 3 Chongqing Key Laboratory of Heterogeneous Material Mechanics, Chongqing University, \\ Chongqing 400044, China \\ 4 School of Mechanical Engineering, Tianjin University, Tianjin 300350, China \\ * Correspondence: gsshui@bjtu.edu.cn (G.S.); yswang@bjtu.edu.cn (Y.-S.W.)
}

Received: 10 December 2019; Accepted: 19 January 2020; Published: 21 January 2020

Featured Application: In this paper, the propagation of non-linear Lamb waves in a tri-layer adhesive structure model with micro-cracks distributing in a random way is systematically investigated using the described numerical simulation method. This study is expected to provide a deeper understanding of the characterization of the damage of the adhesive layer with micro-cracks for adhesive joints by effectively employing the non-linear Lamb waves.

\begin{abstract}
With the advantages of uniform stress transfer and weight reduction, adhesive joints are widely used in engineering. The propagation of non-linear Lamb waves in an adhesive joint with micro-cracks distributing in a random way is systematically investigated by using the numerical simulation method in this paper. A finite element model of the tri-layer adhesive structure with micro-cracks distributing randomly is established, and the Lamb wave mode pair with a matching condition of the phase velocity is chosen to examine the interaction of the micro-cracks with Lamb waves. The results show that the micro-cracks within the adhesive layer will lead to the generation of second harmonics. We also find that the Acoustic Non-linearity Parameters (ANP) increase with the propagation distance in the micro-crack damage zone and the density of the micro-cracks. However, ANPs are less concerned with the friction coefficients of the surface of micro-cracks. This numerical research reveals that non-linear Lamb waves can be employed to effectively characterize the micro-cracks related damages within an adhesive joint.
\end{abstract}

Keywords: non-linear Lamb wave; micro-crack damage; adhesive joint; acoustic non-linearity parameters

\section{Introduction}

Adhesively-bonded structures are widely employed in industries because of their inherent nature of providing uniform stress transfer, high fatigue resistance, and the reduction in structural weight [1]. Under in-service conditions, defects such as voids, micro-cracks, disbonding and kissing bonds often occur in adhesive joints due to fatigue loading and environmental corrosion. For example, micro-cracks are always the precursor to structural failures, which can lead to serious consequences and huge costs in engineering without timely and efficient detection. Thus, the methods of evaluating the quality of bonded joints have been broadly investigated for the purpose of structural safety, which include non-destructive evaluation (NDE) techniques-based ultrasonic testing, acoustic emission, and acoustic elasticity technology [2]. 
As one of the most powerful non-destructive techniques, the ultrasonic detection method has been applied extensively in detecting such defects as voids, cracks and fractures in structural materials. It is based on the principle of linear acoustic physics, including transmission, reflection, scattering, and absorption of the acoustic energy. For example, Lugovtsova et al. [3] analyzed the propagation of guided waves in a plate consisting of an isotropic aluminium layer bonded to an anisotropic laminate made of carbon fibre reinforced plastics. Their results show that most of the wave energy can be concentrated in a certain layer depending on the mode used, and damage presenting in this layer can be detected. However, it is not effective for linear ultrasonic evaluation methods to characterize the early material degradation when the above-mentioned defects are tiny. Particularly, it is maybe impossible to detect the micro-cracks in closing when the ultrasonic testing technology based on linear acoustic physics is employed. Transmissions, reflections and scatterings could not be discerned as the ultrasonic wave propagates through the micro-cracks in closing [4]. For adhesive bonded structures, studies also show that linear Lamb wave is not sensitive to the mechanical property of the adhesive layer, although it is strongly sensitive to the thicknesses and mechanical properties of other plates in the adhesive structure $[5,6]$.

In recent years, the non-linear ultrasonic technique has received attention for the reason that it is highly sensitive to material micro-structures whose dimensions are much smaller than the wavelength of an ultrasonic wave [7-12]. Li et al. [13,14] conducted studies on monitoring the thermal aging of aluminum alloy and detecting the delamination of lined anti-corrosion pipes using non-linear acoustics, which demonstrate the sensitivity of the non-linear ultrasonic technique to the damage. Compared with the linear ultrasonic technique, it is becoming a quite promising tool in the nondestructive characterization of the degrees of damages which are related to the micro-cracks [15-18]. Non-linear ultrasonic technique is based on the non-linear interactions of the ultrasonic wave with the microstructures of materials, such as dislocation, precipitation, and micro-cracks. According to different non-linear effects, sub-harmonic technology, acoustoelasticity, harmonic generation, and wave mixing are common methods for measuring the non-linearity in solids. Both experimental investigations and theoretical studies show that these techniques can detect and evaluate micro damages effectively, and the sensitivity is much higher than traditional linear ultrasonic techniques [19-22].

Non-linear Lamb waves are widely acknowledged as one of the most promising means for quantitatively evaluating the material damages because of its long propagation distance in plate-like structures $[23,24]$. Quite a few studies have shown that the nondestructive ultrasonic evaluation method employing non-linear Lamb waves is capable of characterizing early material degradations and micro-cracks initiation by examining the non-linear effect which is exhibited from the ultrasonic waves [25-30]. Interaction between the elastic wave and micro-cracks with the clapping mechanism has been adopted to explain the generation of higher harmonics of non-linear Lamb waves. As the propagating elastic wave meets a micro-crack within the material, the micro-crack may open or close under the action of tension or compression stress [31,32]. The Lamb wave can propagate through the micro-crack during its compressive period, but not during the tensile period. In this way, the non-linear wave with higher harmonic components is thus generated because of the bi-linear response of the micro-crack under the action of compressive and tensile stresses. In application it is quite a challenge, however, to detect the higher harmonic components of the Lamb wave because of its dispersive property.

Theoretical and experimental studies have been conducted extensively based on the generation of higher harmonics of the non-linear Lamb-wave. For example, Liu et al. [33] analyzed the selection of the primary Rayleigh-Lamb ultrasonic wave mode that generated cumulative second harmonics in a homogeneous isotropic plate using theoretical modeling. Deng et al. [34] experimentally investigated the accumulative effect of the generated second harmonic wave, and their results verified that the generated second harmonic wave increased in a linear way with the increasing propagation distances. Pruell et al. [35] used the non-linear guided waves to evaluate the plasticity driven material damage in a metal plate, and proved that the accumulated plasticity, which caused an increase in the generation 
of second harmonics, can also be measured with Lamb waves. Liu et al. [36] investigated the symmetry properties of second harmonic Lamb waves using an experimental method and proved that the generation of second harmonics will not happen in anti-symmetric Lamb wave modes. Li et al. [37] investigated the second harmonics generation of guided wave propagation in an isotropic and stress-free elastic pipe, and verified that the second harmonics in the pipe have a cumulative effect with the propagation distance. Deng et al. [38] investigated the influences of the interfacial properties on the second harmonic generation of the Lamb waves in layered planar structures. Their results indicated that the efficiency of second harmonic generation is closely dependent on the interfacial properties. Zhao et al. [39] investigated the second harmonic generation in composites and indicated that the phase matching and nonzero power flux criteria are necessary conditions for cumulative second harmonic existence, and only symmetric modes can be generated as cumulative second harmonics for symmetric composite laminates, regardless of the propagation direction of the primary mode.

Because of the presence of interfaces, the waves in the multilayered structures will be refracted and reflected many times, making the propagation characteristic of the Lamb waves more complicated. The numerical simulation method, therefore, is also an effective tool to investigate the process and mechanism of the higher harmonic generation of the Lamb waves for materials with damage. Zhao et al. [40] investigated the generation of non-linear Lamb wave propagation in a thin plate with micro-cracks distributing in a random way. The results showed that the micro-cracks, which lead to the generation of second harmonics, can be applied to simulate the early micro damages in structures. Liu et al. [41] examined the strongly cumulative second harmonic due to the Lamb and SH wave mode interactions using finite element modeling, and the results showed that only the symmetric Lamb mode of the secondary wave field can be cumulative. Zhu et al. [42] performed finite element simulations on the necessity of phase velocity matching and group velocity matching. Their results verified that matching of the phase velocity is necessary for generating the non-linear Lamb waves with cumulative second harmonics, while the matching of the group velocity is not a necessary condition.

In this paper, the propagation of non-linear Lamb waves in a tri-layer adhesive structure model with micro-cracks distributing in a random way is systematically investigated using the numerical simulation method. Based on the dispersion curves of the tri-layer structure, the Lamb wave mode pair matching the phase velocity is chosen to investigate the interactions of the micro-cracks and the Lamb waves. The relationships between the acoustic non-linear parameters and the propagating distances within the micro-crack damage areas, as well as other factors related to the micro-cracks, such as crack density and the friction coefficients of the micro-crack surfaces, are obtained quantitatively. This study is expected to provide a deeper understanding for the characterization of the damage of the adhesive layer with micro-cracks for the adhesive joints by effectively employing the non-linear Lamb waves.

\section{Dispersion Curves of Lamb Waves}

The schematic of a tri-layer adhesive structure composed of two aluminum layers and an adhesive layer is described in Figure 1. Two aluminum plates are joined by an adhesive layer of epoxy resin. The thicknesses of the aluminum layer and adhesive layer are $h_{1}$ and $h_{2}$, respectively. It is assumed that the material of each layer is isotropic, elastic and homogenous. The Lamb waves propagate within the tri-layer adhesive structure along the positive $x$-axis and the wave polarization is along the $z$-axis. The displacements of particles, therefore, occur only in the $x z$ plane for the Lamb waves.

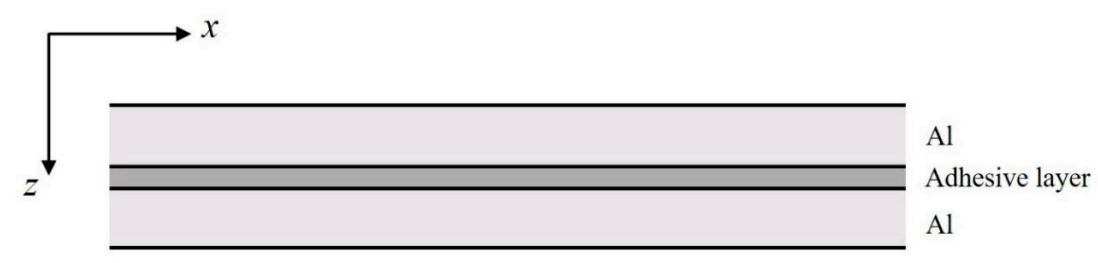

Figure 1. The schematic of the tri-layer adhesive structure. 
For the tri-layer adhesive structure, the state vector $V_{U}^{(j)}$ of the upper surface of the $j$-th layer can be expressed as:

$$
V_{U}^{(j)}=\left(\begin{array}{c}
u_{u}^{(j)} \\
w_{U}^{(j)} \\
\sigma_{z U}^{(j)} \\
\sigma_{z x U}^{z j}
\end{array}\right)=P_{U}^{(j)} e^{i k(x-c t)}\left(\begin{array}{c}
A^{(j)} \\
B^{(j)} \\
C^{(j)} \\
D^{(j)}
\end{array}\right)
$$

where $u_{U}^{(j)}, w_{U}^{(j)}, \sigma_{z U}^{(j)}$ and $\sigma_{z x U}^{(j)}$ are the displacements and stresses in $x$ - and $z$-directions, respectively. The subscript $U$ refers to the upper surface; $P_{U}^{(j)}$ is the amplitude of the state vector; $A^{(j)}, B^{(j)}, C^{(j)}$ and $D^{(j)}$ are constants of the $j$-th layer. For this tri-layer adhesive structure, the relation between the state vectors of the upper and lower surfaces for each layer can be expressed as:

$$
V_{L}^{(j)}=T^{(j)} V_{U}^{(j)}
$$

where $V_{L}^{(j)}$ is the state vector of the lower surface of the $j$-th layer; $T^{(j)}$ is the transfer matrix of the $j$-th layer. For the whole tri-layer structure, therefore, state vectors of the upper and lower surfaces can be related as:

$$
\left(\begin{array}{c}
u_{L} \\
w_{L} \\
\sigma_{z L} \\
\sigma_{z x L}
\end{array}\right)=\mathrm{T}\left(\begin{array}{c}
u_{U} \\
w_{U} \\
\sigma_{z U} \\
\sigma_{z x U}
\end{array}\right)
$$

where $T=T^{(1)} T^{(2)} T^{(3)}$.

Considering the free surface condition of the upper and lower surface for the tri-layer structure, the following equation can be obtained, according to Equations (1)-(3):

$$
\left[\begin{array}{ll}
T(3,1) & T(3,2) \\
T(4,1) & T(4,2)
\end{array}\right]\left(\begin{array}{c}
u_{U} \\
w_{U}
\end{array}\right)=\left(\begin{array}{l}
0 \\
0
\end{array}\right)
$$

To avoid the trivial solution of Equation (4), the following expression must be satisfied:

$$
\operatorname{det}\left[\begin{array}{ll}
T(3,1) & T(3,2) \\
T(4,1) & T(4,2)
\end{array}\right]=0
$$

Equation (5) is the dispersion equation of the tri-layer adhesive structure. By solving this equation numerically, the dispersion curves can be plotted, as shown in Figure 2. Mode conversions for the tri-layer adhesive structure can be clearly found, differing from the dispersion curves of a single plate. For the dispersion curves of the tri-layer adhesively-jointed structure, the phase velocities converge to the transversal wave velocity of the adhesive layer.

To generate the accumulative second harmonics of Lamb waves, the Lamb wave mode should satisfy the conditions of nonzero power flux from the fundamental to the second harmonic wave and phase velocity matching [43]. Considering that the lower order modes carry more energy than the higher-order modes for the Lamb waves propagating within the tri-layer adhesive structure, the first symmetric mode pair of $S_{1}$ and $S_{2}$ are chosen as the fundamental and second harmonic waves, respectively. As shown in Figure 2a, when the frequency of the fundamental Lamb waves (Mode $S_{1}$ ) is selected as $0.52 \mathrm{MHz}$, accumulative second harmonic waves (Mode $\mathrm{S}_{2}$ ) with the frequency of $1.04 \mathrm{MHz}$ can be generated with the same phase velocity. Although the matching condition of the group velocity is not satisfied according to Figure $2 b$, the second harmonic Lamb waves will still accumulate with the propagation distance if the conditions of nonzero power flux from the fundamental wave to the second harmonic wave and phase velocity matching are satisfied [44]. 


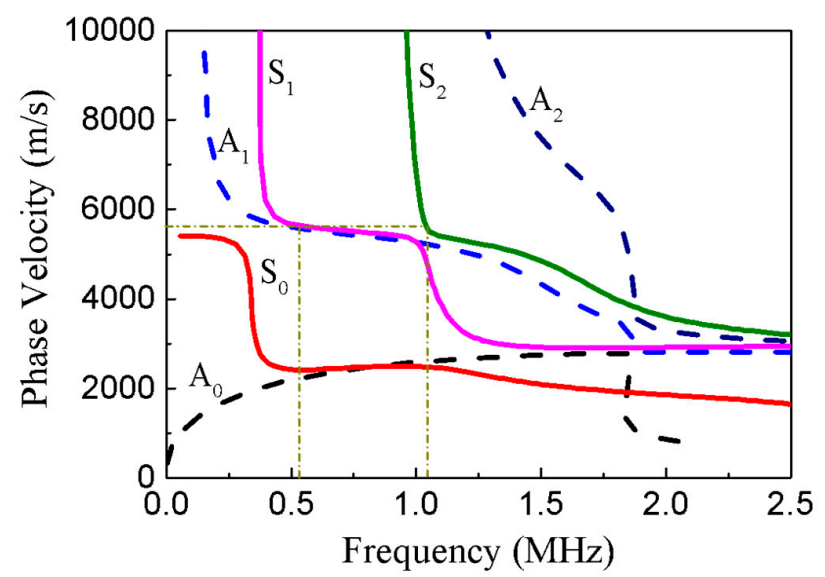

(a)

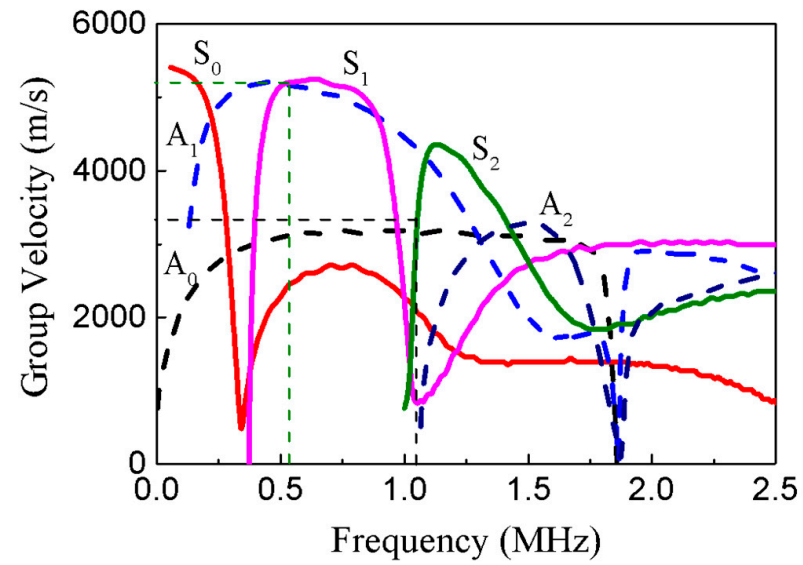

(b)

Figure 2. The dispersion curve of the tri-layer adhesive structure. (a) Phase velocity versus the frequency and (b) Group velocity versus the frequency. The solid lines represent the symmetric modes; the dashed lines represent the anti-symmetric modes.

\section{Numerical Simulations}

It is quite challenging to get the analytic non-linear responses of Lamb waves propagating along the tri-layer adhesive with randomly distributed micro-cracks in the adhesive layer. Therefore, numerical simulations are employed instead in this work. The commercial software Abaqus/EXPLICIT is used, which is employed by the central difference method and conditionally stable. A finite element model in two dimensions is set up for the tri-layer adhesive structure with micro-cracks distributing in a random way within the adhesive layer.

As shown in Figure 3, the middle part of the adhesive layer with a length of $L$ is the area with micro-crack damage. A dynamical displacement disturbance is exerted on the left side of the model in order to generate the Lamb wave in this tri-layer adhesive structure. After the interaction of Lamb wave with the micro-cracks distributing randomly in the damaged area, the wave signals propagating toward the direction of positive $x$-axis will be collected at signal detection positions 1 and 2 .

According to the dispersion curves shown in Figure 2, the fundamental mode $S_{1}$ can be excited when the frequency of the dynamical displacement disturbance at the left side is $0.52 \mathrm{MHz}$. This dynamical disturbance is expressed as:

$$
u(x, t)=\frac{1}{2} B\left[1-\cos \left(\frac{2 \pi f t}{15}\right)\right]
$$


where $B$ is the amplitude of the dynamical disturbance with $B=1 \times 10^{-5} \mathrm{~mm}$ in the simulations; and $f$ is the frequency. The Lamb wave propagates in the positive $x$-direction, and interacts with the micro-cracks distributing randomly at the micro-crack damaged area of the adhesive layer. As a result, Lamb waves with the second component $\left(S_{2}\right.$ mode) propagating with the same phase velocity will thus be generated. Most waves will penetrate across a damaged area with a micro-crack because the dimension of the micro-crack is far smaller than the wavelengths of $S_{1}$ and $S_{2}$ mode waves. The transmitted waves will be collected at positions 1 and 2 .

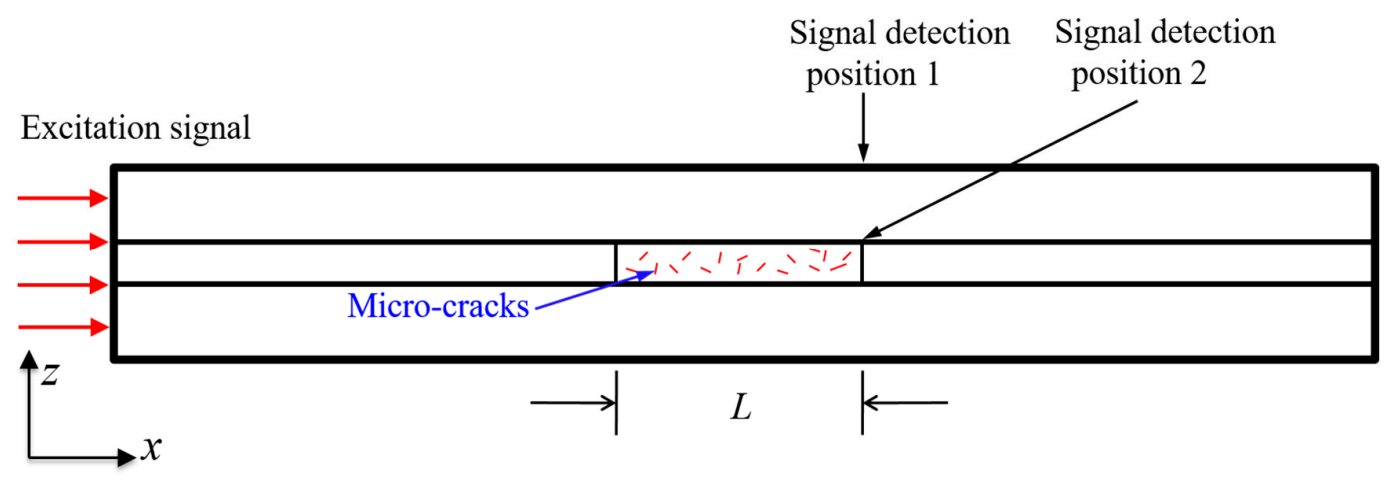

Figure 3. Schematic of the Lamb waves propagating through a region with micro-cracks for the tri-layer adhesive structure.

In the present simulation, linear elastic constitutive models of aluminum and epoxy are employed in FEM models. Material properties of aluminum are $E=72.8 \mathrm{GPa}, \rho=2665 \mathrm{~kg} / \mathrm{m}^{3}, v=0.33$; and those of epoxy are $E=1 \mathrm{GPa}, \rho=1000 \mathrm{~kg} / \mathrm{m}^{3}$ and $v=0.45$. The dimension of the model is $500 \mathrm{~mm} \times 3.3 \mathrm{~mm}$, where the thickness of the aluminum plate is $1.5 \mathrm{~mm}$ and that of the adhesive layer is $0.3 \mathrm{~mm}$. The length of the micro-crack damage zone is $L$, which ranges from $4-16 \mathrm{~mm}$.

The micro-cracks are assumed to be distributed in the space with an area $A$ at the micro-crack region of the adhesive layer. In the simulation, we assume that the length of each micro-crack is $2 a$. The crack density is defined using a dimensionless parameter as:

$$
c=\frac{N a^{2}}{A}
$$

where $N$ is the number of micro-cracks. The center and direction of the micro-crack, which are variables affecting the random distribution of the micro-cracks, can be represented by the function of the probability density. In this numerical simulation, the variable of the function is uniformly random.

Consider the surfaces of the micro-cracks as contacting surfaces which will separate from each other when Lamb waves pass through them while being unable to interpenetrate into each other. A contact model is thus employed to represent the clapping and slipping behavior of the micro-crack. To account for the influence of the surface friction to the Lamb wave propagation, the Coulomb friction law is employed in modeling the relative sliding of the micro-crack surfaces. The tensile stress exerting on the surfaces of the micro-crack is expressed as [45]:

$$
\mathbf{q}=\sigma_{0} \mathbf{n}+\left[\tau_{0}+\mu \sigma_{0} \mathrm{H}\left(-\sigma_{0}\right)\right] \mathrm{H}\left[\tau_{0}+\mu \sigma_{0} \mathrm{H}\left(-\sigma_{0}\right)\right] \mathbf{s}
$$

where $\sigma_{0}$ is the applied normal stress; $\tau_{0}$ is the shear stress on the surfaces of the micro-cracks; $\mu$ is the friction coefficient of the contacting surface; $\mathrm{H}(\bullet)$ is the Heaviside step function; $\mathbf{n}$ is a unit vector normal to the crack surface; and $\mathbf{s}$ is in the plane of the crack. In the numerical simulations, the built-in contact models of the Abaqus software are adopted, which are the hard contact law of the normal behavior and the Coulomb friction law of the tangential behavior.

To guarantee enough accuracy and stability during the numerical simulation, we will use more than 20 elements within the smallest wavelength of all the Lamb wave modes. The largest element 
sizes, therefore, for the aluminum layer and the adhesive layer are set to be $0.2 \mathrm{~mm}$ and $0.08 \mathrm{~mm}$, respectively. Six elements are employed when modeling the surface of the micro-crack. The four-node plane strain (CPE4R) element is employed to construct the FEM model. As to the time step for the numerical simulation, it should satisfy the condition that the Lamb wave does not travel farther than the size of the smallest element within a time step. The stable time step is chosen to be $\Delta t=5 \times 10^{-10} \mathrm{~s}$ when the numerical accuracy and efficiency are also taken into consideration. Shown in Figure 4 is the finite element modeling and mesh of the region with micro-cracks distributing in a random way.

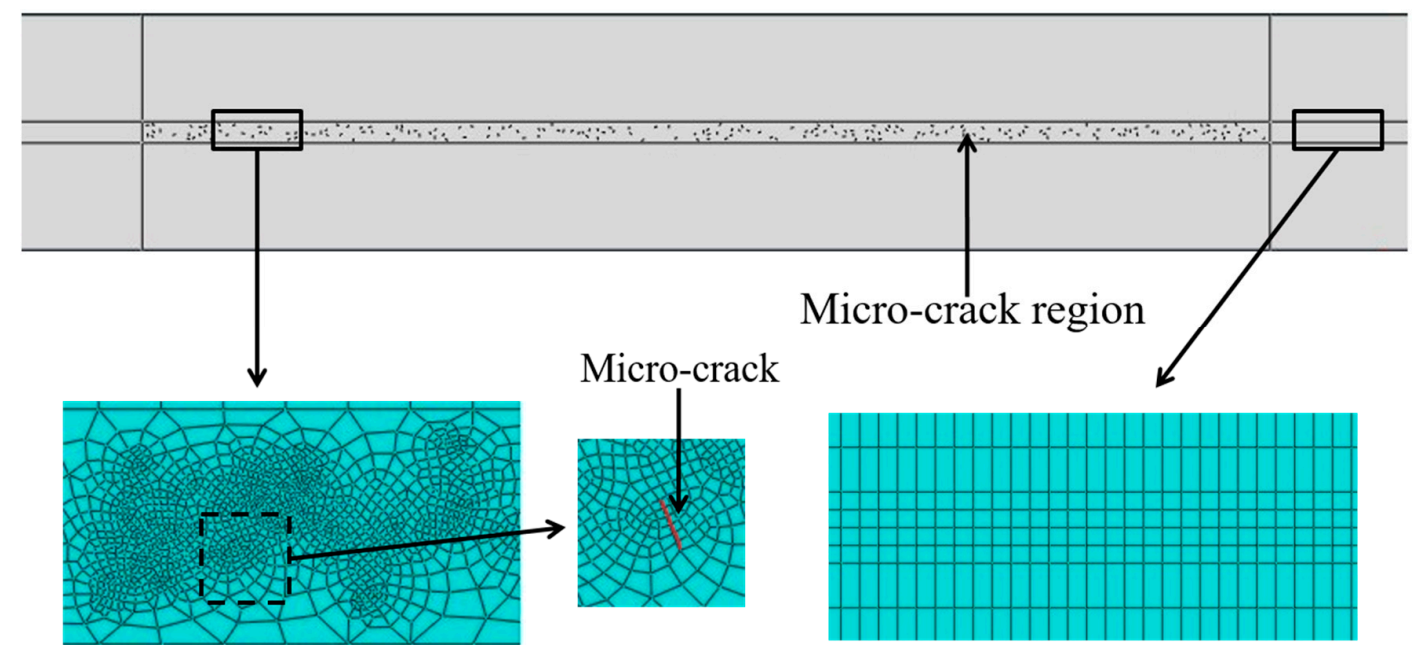

Figure 4. The FEM model of the micro-crack damage region: The distribution of micro-cracks and the finite element mesh.

The non-linearity of acoustics caused by the contacting surface of the crack is a clapping effect of the surface and non-linear stress-strain behavior inherent in the contact, which is different from the theory of classical non-linearity. Some theoretical models have been reported for explaining the contacting acoustic non-linearity; for example, the bi-linear stiffness models and hysteretic models [46,47]. Zhao et al. [48] further investigated the bi-linear stiffness models when different effective tensile and compressive moduli of the local micro-crack region are taken into consideration. For non-linear ultrasonic wave propagating within the material with micro-cracks, they suggested that the Acoustic Non-linearity Parameter (ANP) should be defined as:

$$
\beta^{\prime}=\frac{M_{2}}{M_{1}}
$$

where $\beta^{\prime}$ is the ANP that has a close relationship to the density and friction coefficient of the micro-crack; $M_{1}$ and $M_{2}$ are the magnitudes of fundamental and second harmonics of non-linear Lamb wave, respectively. In the following numerical simulations, Equation (9) will be employed in investigating the relationship between the ANP and the micro-crack.

Moreover, since the micro-cracks in the model are randomly distributed, numerical simulations were conducted repeatedly to eliminate the random effect of distribution. The averaged ANP based on the results of numerous finite element models versus the number of simulations is shown in Figure 5. Note that the average ANPs are based on the amplitudes of the fundamental and second harmonic waves collected in the $x$ - and $z$-direction. It is clearly seen that when the number of simulations reaches 10 , the average ANP tends to be stable. Thus, the ANPs in the following simulations are all the average values of 25 models with micro-cracks distributing randomly. 


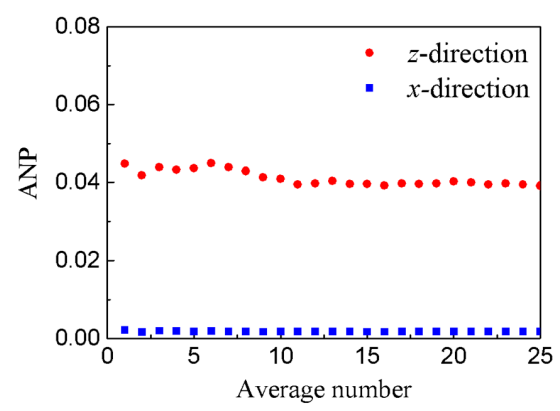

Figure 5. The average Acoustic Non-linearity ParameterANP versus the average number of simulations based on the displacement collected in the $x$-direction and $z$-direction using the models.

\section{Results and Discussions}

Figure 6 shows the displacements contour of the Lamb wave which propagates in the tri-layer adhesive structure with micro-cracks distributing in a random way within the adhesive layer. $\mathrm{U}_{1}$ and $\mathrm{U}_{2}$ are the displacements in the $x$ - and $z$-directions, respectively. It is clear that the length of the micro-crack is much shorter than the wavelength of the Lamb wave. In addition, it also shows that there is no big change in the waveform of the Lamb waves after they interact with the micro-cracks when passing through the region with micro-cracks. States of opening and closing can also be observed when the deformed shape is magnified 5000 times for the micro-cracks, as shown in Figure 6a.

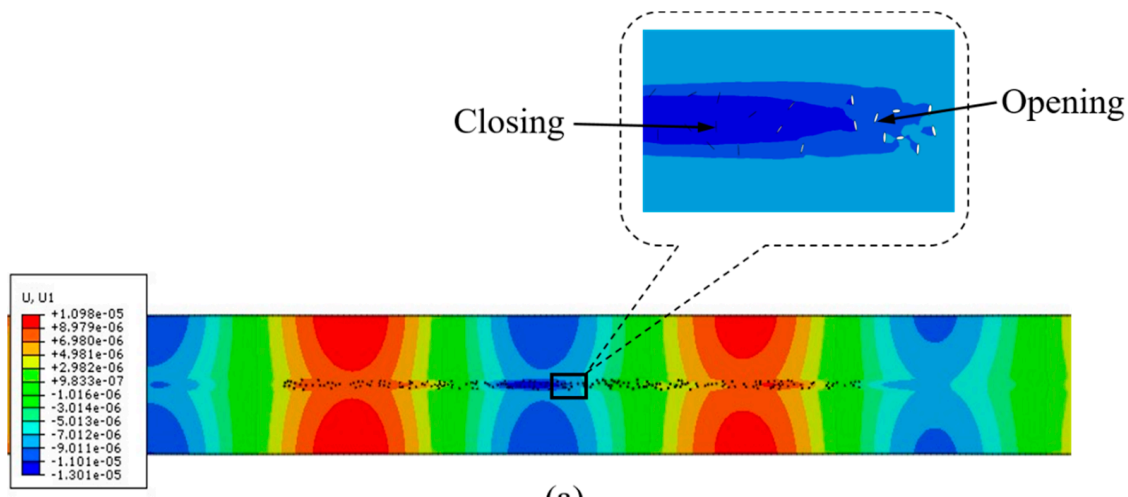

(a)

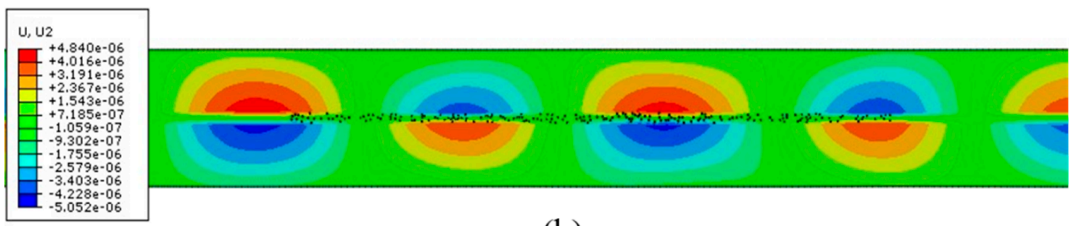

(b)

Figure 6. The Lamb waves propagating in the tri-layer adhesive structure. (a) The displacement contour in the $x$-direction and the 5000-times magnified deformed shapes; (b) the displacement contour in the $z$-direction.

The Lamb waves collected at position 1 (as shown in Figure 3) in the $x$ - and $z$-directions are shown in Figures 7 and 8, respectively. Note that, plots shown in Figures $7 \mathrm{~b}$ and $8 \mathrm{~b}$ are based on the spectrum graph sliced at the fundamental and second harmonic frequency, respectively. In both directions, the $S_{0}$ mode was also generated besides $S_{1}$ and $S_{2}$ modes because the thickness profile of the $S_{0}$ mode is a bit similar to that of the $S_{2}$ mode at the fundamental primary frequency. The solid line represents the waves for the adhesive layer without cracks, while the dashed line represents those with randomly distributed micro-cracks. As we can see, the time-domain signals for both cases, with and without micro-cracks, are almost overlapped, as shown in Figures 7a and 8a. Magnitudes of fundamental harmonics and second harmonics (collected at position 1) versus the propagation distance 
are shown in Figure 9. We can see that magnitudes of fundamental harmonics vary slightly with the propagation distance, while there is accumulative increase of the magnitude of second harmonics with the propagation distance. It means that we can neglect the influence of the micro-crack on the propagation of fundamental Lamb waves. According to the frequency domain plot shown in Figures $7 \mathrm{~b}$ and $8 \mathrm{~b}$, however, the dashed line contains second harmonic waves for time-domain signals in either the $x$ - or $z$-direction when there are micro-cracks within the adhesive layer, which demonstrates that the micro-cracks lead to the generation of second harmonic Lamb waves. The minimum propagation distance in our simulation is $4 \mathrm{~mm}$; we can see that there are second harmonics at this distance.

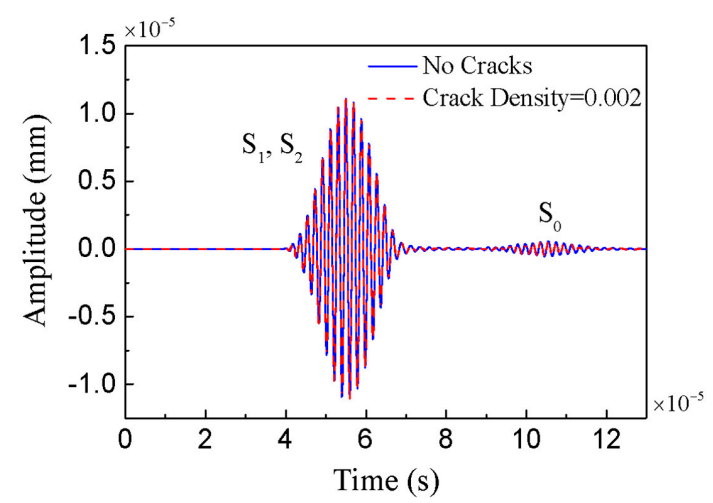

(a)

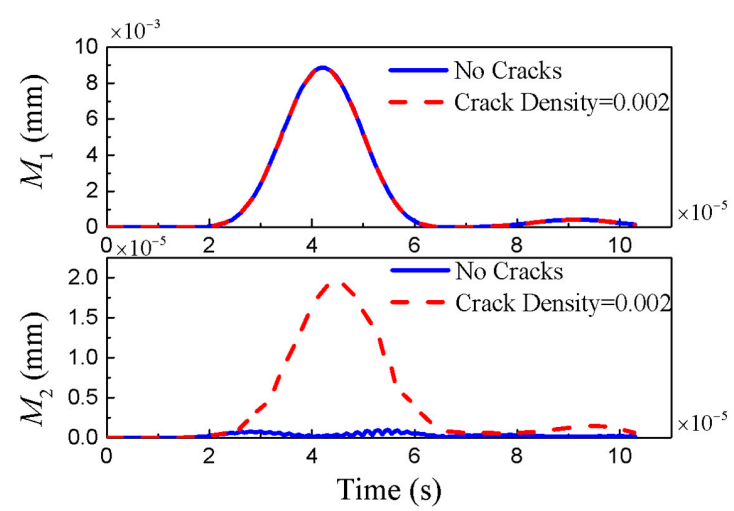

(b)

Figure 7. Lamb waves collected at position 1 in the $x$-direction. (a) In the time domain; (b) the spectrum graph sliced at the fundamental frequency $0.52 \mathrm{MHz}$ and second harmonic frequency $1.04 \mathrm{MHz}$ $(c=0.002, \mu=0.3)$.

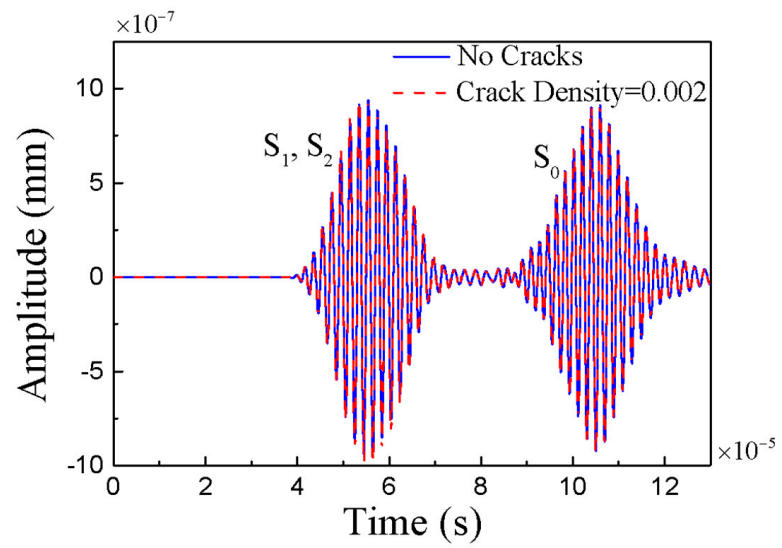

(a)

Figure 8. Cont. 


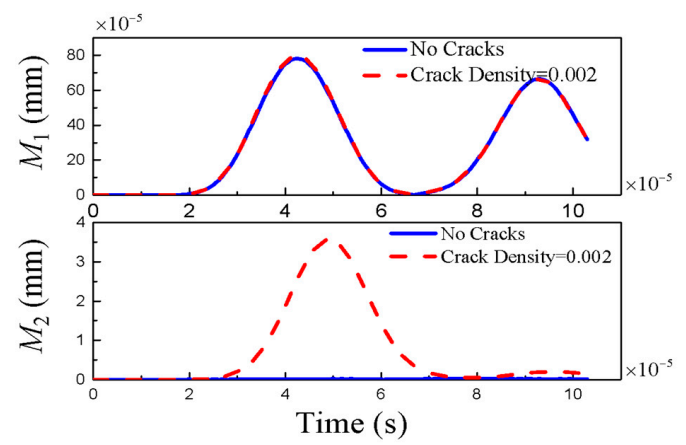

(b)

Figure 8. Lamb waves collected at position 1 in the $z$-direction. (a) The time domain; (b) the spectrum graph sliced at the fundamental frequency $0.52 \mathrm{MHz}$ and second harmonic frequency $1.04 \mathrm{MHz}$ $(c=0.002, \mu=0.3)$.

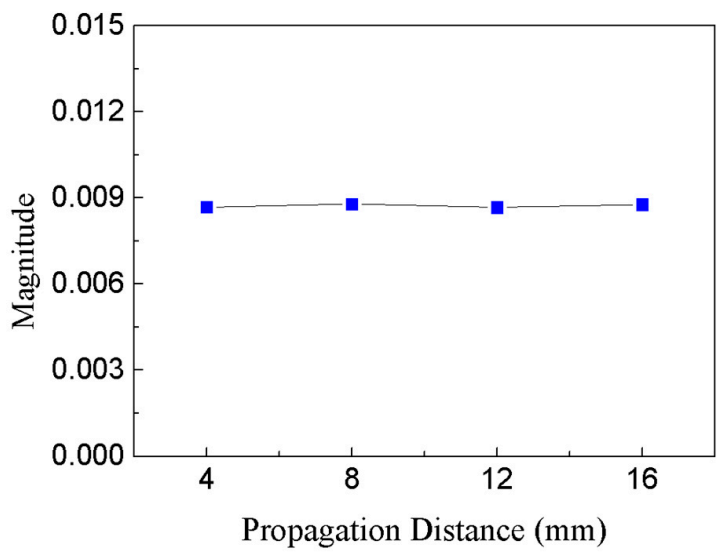

(a)

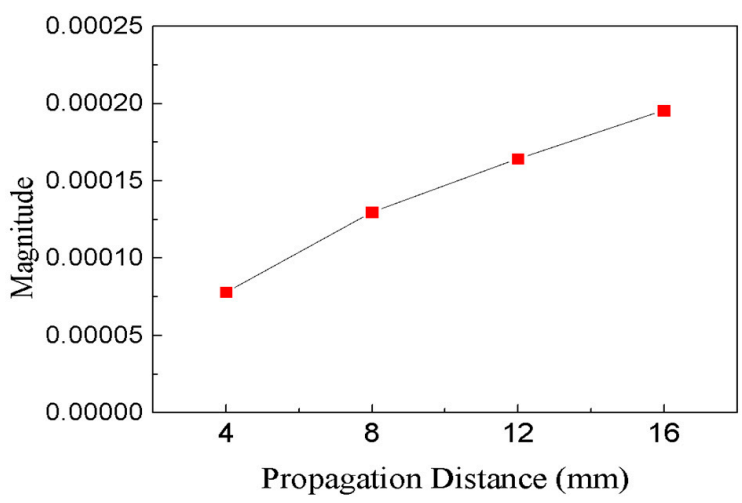

(b)

Figure 9. Magnitudes of (a) fundamental harmonics and (b) second harmonics (collected at position 1) versus the propagation distance.

Figures 10 and 11 show the ANPs versus the propagation distances of the Lamb wave signals collected at position 1 and position 2, respectively. To validate the numerical simulation, ANPs without randomly distributed micro-cracks are also provided. It is clear that the ANPs in the healthy state are not only much smaller than with randomly distributed micro-cracks, but also change little with the propagation distances. This indicates that it is the randomly distributed micro-cracks that lead to the generation of second harmonic waves. In Figure 3, the length of the damage zone with micro-cracks varies from 4-16 mm. Note that the signals are collected at the end of the micro-crack zone; the length of micro-crack zone, therefore, is the propagation distance of the Lamb waves. As can be seen, the 
ANPs increase when the distances of the Lamb wave propagating in the micro-cracks damage zone increase, except for the signals collected at position 1 in the $z$-direction. It demonstrates that the second harmonic Lamb waves show a property of accumulation with the propagation distance. Note that there is an exception for the ANP collected at position 1 in the $z$-direction. Investigations have demonstrated that the magnitude of the second harmonics would increase in a linear way with the propagating distances of non-linear Lamb waves when the phase velocities of the fundamental and second components are equal [49]. If they are not equal to each other, the magnitude of the second harmonics will then vary in the form of a sinusoidal curve with increasing propagation distances. Although we try to excite the fundamental and second harmonics of Lamb waves with the same phase velocity, the existence of the micro-crack, however, will lead to small deviations of the phase velocities when the non-linear Lamb waves propagate in the region with micro-cracks. The ANPs, therefore, do not always exhibit a linear relationship with the propagation distance, particularly in the region of micro-cracks. This effect is more obvious for the ANP collected at position 1 in the $z$-direction.

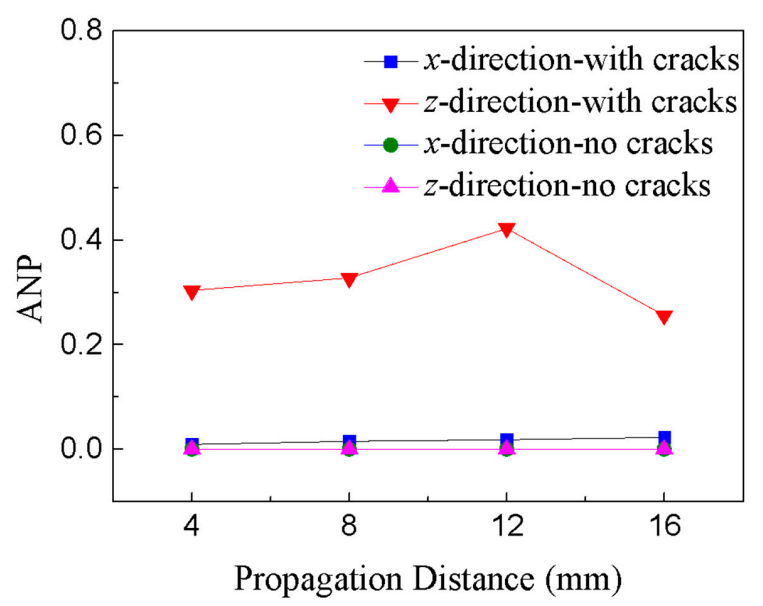

Figure 10. ANP (collected at position 1) versus the propagation distance.

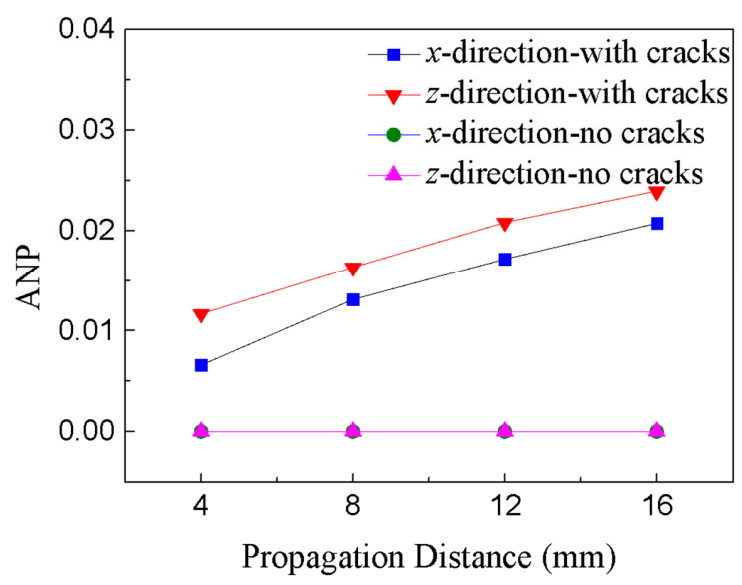

Figure 11. ANP (collected at position 2) versus the propagation distance.

Furthermore, Figures 10 and 11 show that the ANPs in the $z$-direction are bigger than those in the $x$-direction, which indicates that the non-linear effect in the $z$-direction is stronger. Particularly, the ANPs in the $z$-direction from the signals collected at position 1 are much bigger than that at position 2 . This phenomenon can be explained using the displacement field of the excited second harmonic Lamb waves, as shown in Figure 12. It is clear that the normalized displacement in z-direction is bigger at the surface (position 1) than that at the adhesive layer (position 2). This fact indicates that it is more effective to evaluate the non-linearity of the adhesive structure just by employing the $z$-component of the Lamb waves at the surface, which is easy to realize in practice. 


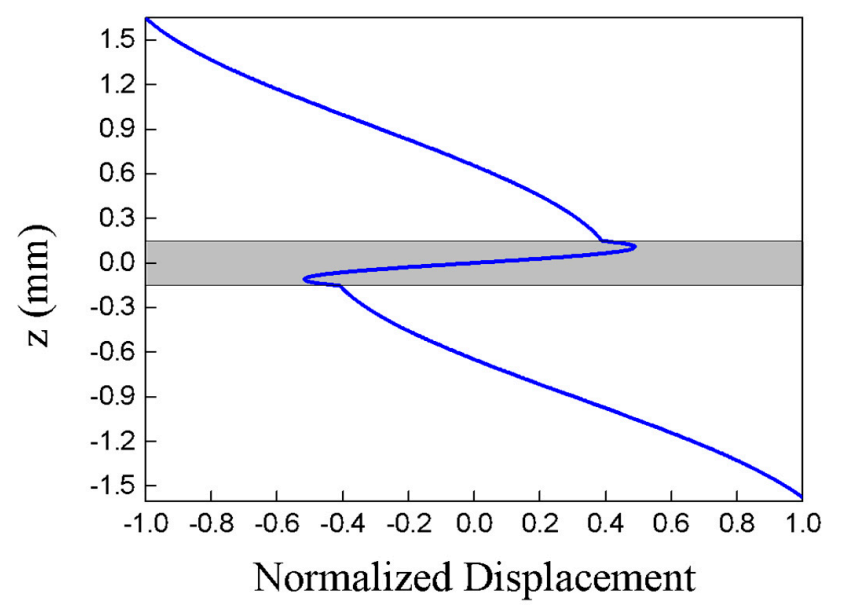

Figure 12. The displacement field of the second harmonic Lamb waves.

As a quantitative indicator for the micro-cracks, the crack density, as defined in Equation (7), is closely related to the number and length of the cracks within the adhesive layer. Figure 13 shows the ANP as a function the micro-crack number when other factors, such as the crack length and friction coefficient, remain unchanged. It is demonstrated that there is a linear relationship between the ANP and the micro-crack number, both in the $x$ - and $z$-directions. Because the ANP based on the signals collected at position 1 is more sensitive to the micro-crack related damage, here we only consider the signals collected at position 1. Figure 14 plots the relationship between the ANP and micro-crack length. It is clear that there is a linear relationship between them. Furthermore, it also shows that the ANPs are more sensitive to the change of the micro-crack length when other factors are kept constant. The ANP versus the micro-crack density with increasing micro-crack number and increasing radius is shown in Figure 15. Considering that the micro-cracks are distributing randomly for the finite element modeling, it is still demonstrated that the results based on these two cases are basically consistent. At the same time, when such factors as the frequency of the exciting signals and friction coefficient of the micro-crack surface keep constant, the ANPs also increase in a linear way with the increasing micro-crack density. Meanwhile, the ANPs in the $z$-direction are obviously higher than those in the $x$-direction for all the cases. In engineering applications, it is possible to evaluate the micro-crack induced damage of an adhesive layer by the ANP of the $z$-component at this adhesive surface.

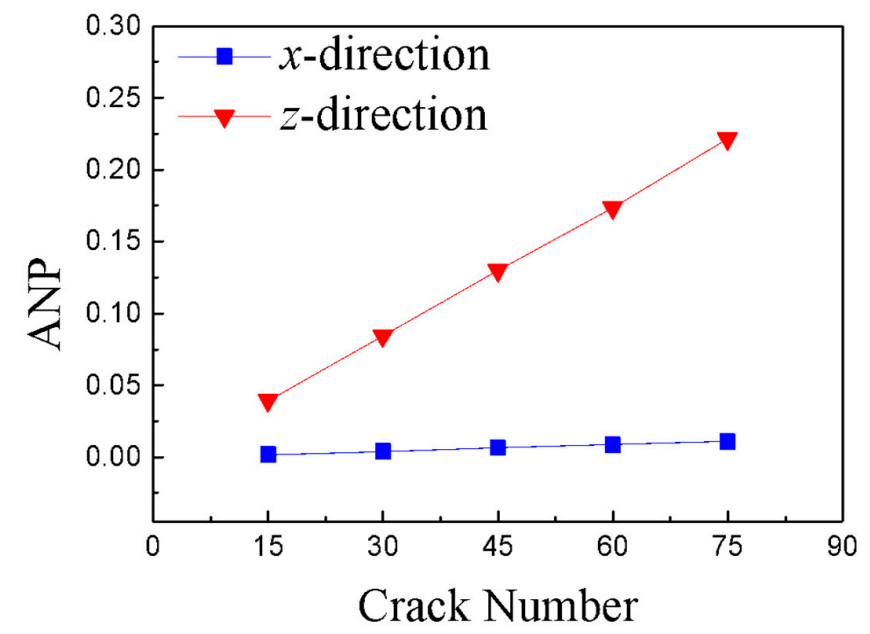

Figure 13. ANP versus crack number $(\mu=0.3)$. 


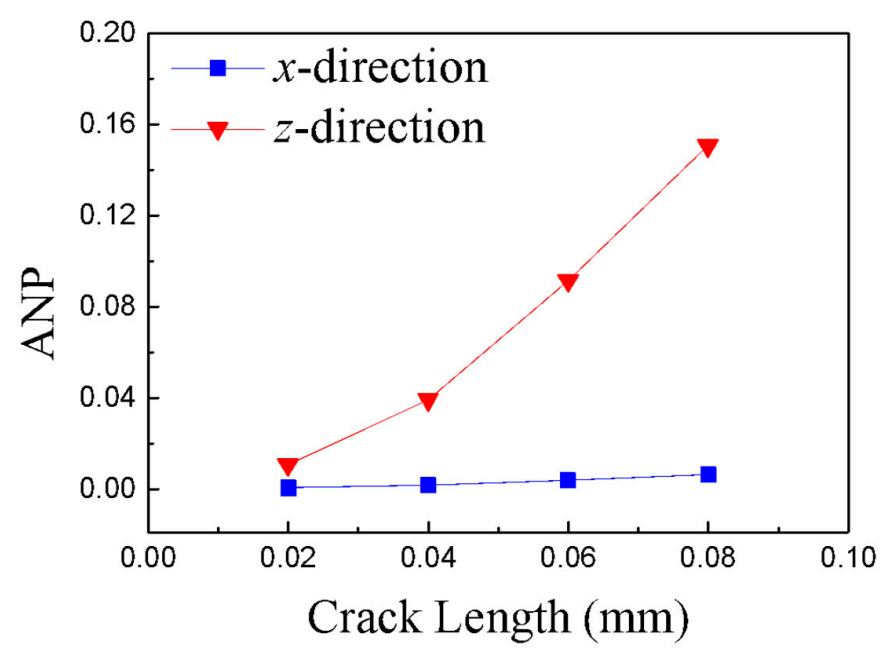

Figure 14. ANP versus crack length $(\mu=0.3)$.

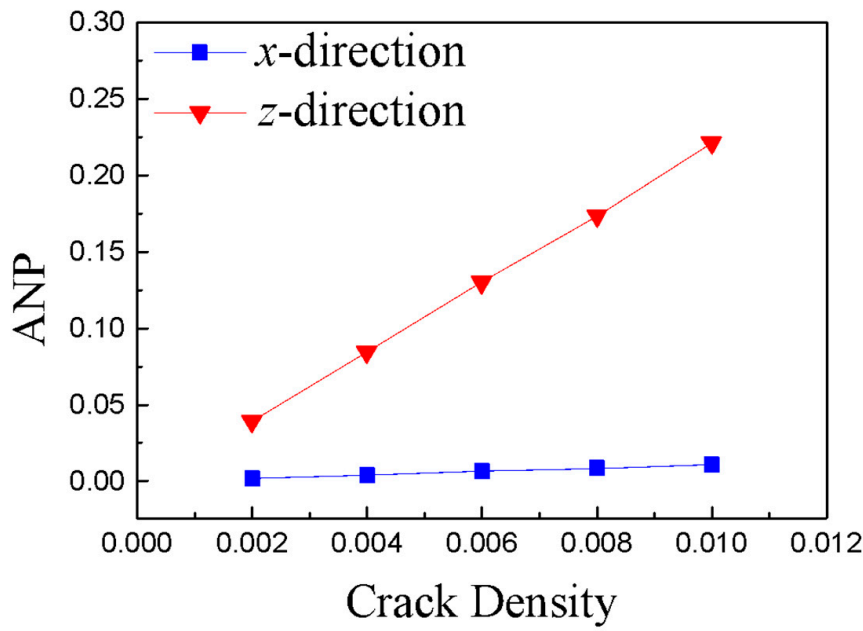

(a)

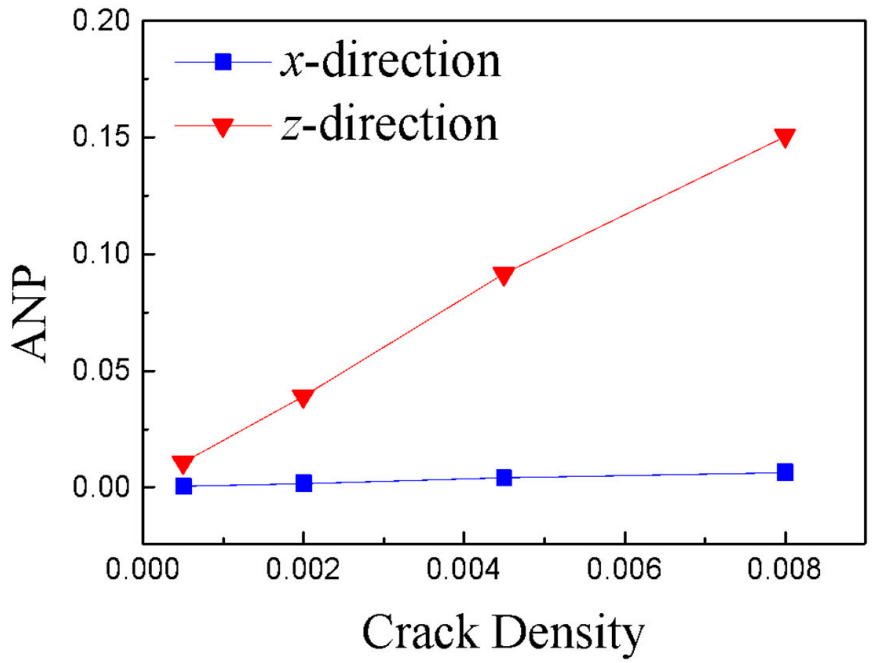

(b)

Figure 15. ANP versus crack density with (a) increasing crack number only and (b) increasing radius only $(\mu=0.3)$.

In contrast to the classical non-linear acoustics, the friction coefficient between the micro-crack surfaces can also affect the stress in the structure but may have an influence on the nonlinear effect of 
the Lamb waves. The Coulomb law is employed in calculating the action between the micro-crack surfaces where there is friction, as defined in Equation (8). Figure 16 shows the ANP (collected at position 1) as a function of the friction coefficient of micro-crack surface. As can be seen, the ANPs change within a quite small range when the friction coefficient varies from 0 to 0.3 . It means that the ANPs are slightly related to the friction coefficient of micro-crack surfaces. The reason is that the micro-crack surfaces do not slide obviously from each other under the action of the transmitted Lamb waves when they penetrate though the region with micro-cracks. The friction coefficient between the surfaces of the micro-cracks, therefore, nearly has little influence on the non-linear behavior of Lamb waves.

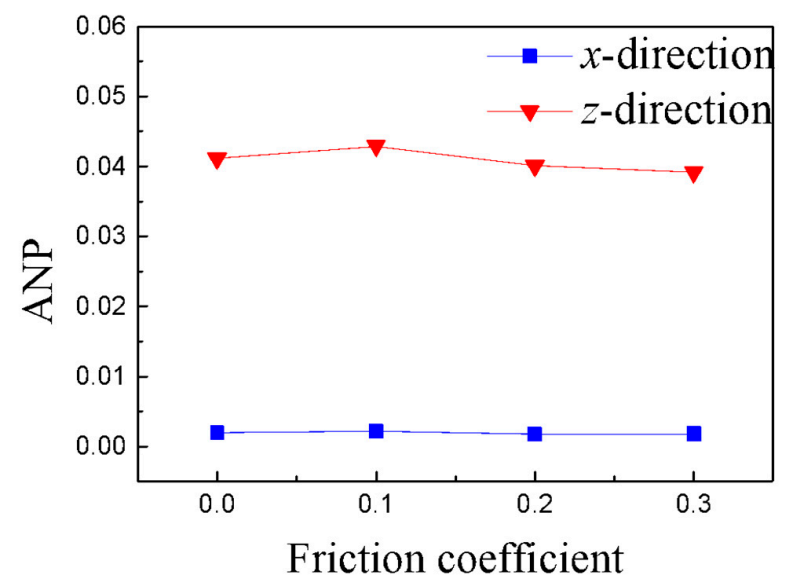

Figure 16. ANP versus friction coefficient $(c=0.002)$.

\section{Conclusions}

In this research, the propagation of nonlinear Lamb waves in an adhesive joint with micro-cracks distributing in a random way is systematically investigated by using the numerical simulation method. Based on the dispersion curves, a tri-layer adhesive structure model with randomly distributed micro-cracks is examined, and the Lamb wave mode pair satisfying phase velocity matching is chosen to investigate the interactions of the micro-cracks and the Lamb waves. The study demonstrates that the higher harmonics will be generated because of the bi-linear stiffness response at the area with micro-cracks under the action of tensile and compressive wave stress. The results show that:

(1) The ANPs increase with the propagating distance of the Lamb wave in the region of micro-cracks. Particularly, the magnitudes of the generated second harmonics in the $z$-direction are bigger than those in the $x$-direction, which indicates that the nonlinear effect in the $z$-direction is stronger.

(2) The ANPs increase linearly with the micro-crack density. Specially, the ANP is more sensitive to the change of the micro crack length.

(3) The ANPs are slightly related to the friction coefficient between the surfaces of micro-cracks.

This investigation numerically demonstrates that the nonlinear Lamb waves can be used effectively in characterizing the micro-crack-induced damages within the adhesive layer of an adhesively bonded joint.

Author Contributions: X.L., G.S. and Y.Z. conceived and designed the simulations; X.L. performed the simulations; X.L. and G.S. wrote the paper; G.S., Y.Z. and Y.-S.W. revised the paper. All authors have read and agreed to the published version of the manuscript.

Funding: This work is supported by the National Natural Science Foundation of China (No. 11472039, No. 11604033) and the Fundamental Research Funds for the Central Universities of China (No. 2019CDXYHK0001).

Conflicts of Interest: The authors declare no conflict of interest. 


\section{References}

1. Rucka, M.; Wojtczak, E.; Lachowicz, J. Damage imaging in Lamb wave-based inspection of adhesive joints. Appl. Sci. 2018, 8, 522. [CrossRef]

2. Liu, Y.; Zhang, M.; Yin, X.; Huang, Z.; Wang, L. Debonding detection of reinforced concrete (RC) beam with near-surface mounted (NSM) pre-stressed carbon fiber reinforced polymer (CFRP) plates using embedded piezoceramic smart aggregates (SAs). Appl. Sci. 2020, 10, 50. [CrossRef]

3. Lugovtsova, Y.; Bulling, J.; Boller, C.; Prager, J. Analysis of Guided Wave Propagation in a Multi-Layered Structure in View of Structural Health Monitoring. Appl. Sci. 2019, 9, 4600. [CrossRef]

4. Brotherhood, C.J.; Drinkwater, B.W.; Dixon, S. The detectability of kissing bonds in adhesive joints using ultrasonic techniques. Ultrasonics 2003, 41, 521-529. [CrossRef]

5. Lowe, M.J.S.; Cawley, P. The Applicability of Plate Wave Techniques for the Inspection of Adhesive and Diffusion Bonded Joints. J. Nondestruct. Eval. 1994, 13, 185-200. [CrossRef]

6. Nagy, P.B.; Adler, L. Nondestructive evaluation of adhesive joints by guided waves. J. Appl. Phys. 1989, 66, 4658-4663. [CrossRef]

7. Metya, A.K.; Tarafder, S.; Balasubramaniam, K. Non-linear Lamb wave mixing for assessing localized deformation. NDT E Int. 2018, 98, 89-94. [CrossRef]

8. Drinkwater, B.W.; Wilcox, P.D. Ultrasonic arrays for non-destructive evaluation: A review. NDT E Int. 2006, 39, 525-541. [CrossRef]

9. Li, F.; Zhao, Y.; Cao, P.; Hu, N. Mixing of ultrasonic Lamb waves in thin plates with quadratic non-linearity. Ultrasonics 2018, 87, 33-43. [CrossRef]

10. Ren, G.; Kim, J.; Jhang, K.Y. Relationship between second- and third-order acoustic non-linear parameters in relative measurement. Ultrasonics 2015, 56, 539-544. [CrossRef]

11. Zhao, Y.; Chen, Z.; Cao, P.; Qiu, Y. Experiment and FEM study of one-way mixing of elastic waves with quadratic non-linearity. NDT E Int. 2015, 72, 33-40. [CrossRef]

12. Shui, G.; Wang, Y.; Gong, F. Evaluation of plastic damage for metallic materials under tensile load using nonlinear longitudinal waves. NDT E Int. 2013, 55, 1-8. [CrossRef]

13. Li, M.; Lomonosov, A.M.; Shen, Z.; Seo, H.; Jhang, K.Y.; Gusev, V.E.; Ni, C. Monitoring of Thermal Aging of Aluminum Alloy via Nonlinear Propagation of Acoustic Pulses Generated and Detected by Lasers. Appl. Sci. 2019, 9, 1191. [CrossRef]

14. Hong, X.; Liu, Y.; Lin, X.; Luo, Z.; He, Z. Nonlinear ultrasonic detection method for delamination damage of lined anti-corrosion pipes using PZT transducers. Appl. Sci. 2018, 8, 2240. [CrossRef]

15. Sohn, H.; Lim, H.J.; DeSimio, M.P.; Brown, K.; Derriso, M. Non-linear ultrasonic wave modulation for online fatigue crack detection. J. Sound Vib. 2014, 333, 1473-1484. [CrossRef]

16. Rjelka, M.; Köhler, B.; Mayer, A. Non-linear effects of micro-cracks on long-wavelength symmetric Lamb waves. Ultrasonics 2018, 90, 98-108. [CrossRef] [PubMed]

17. Shui, G.; Wang, Y.; Huang, P.; Qu, J. Nonlinear ultrasonic evaluation of the fatigue damage of adhesive joints. NDT E Int. 2015, 70, 9-15. [CrossRef]

18. Shui, G.; Song, X.; Xi, J.; Wang, Y. Experimental Characterization of Impact Fatigue Damage in an Adhesive Bonding Using the Second Harmonics. J. Nondestruct. Eval. 2017, 36, 23. [CrossRef]

19. Chen, J.; Xu, Z.; Yu, Y.; Yao, Y. Experimental characterization of granite damage using non-linear ultrasonicte chniques. NDT E Int. 2014, 67, 10-16. [CrossRef]

20. Cantrell, J.H.; Yost, W.T. Non-linear ultrasonic characterization of fatigue microstructures. Int. J. Fatigue 2001, 23, S487-S490. [CrossRef]

21. Balasubramaniam, K.; Valluri, J.S.; Prakash, R.V. Creep damage characterization using a low amplitude non-linear ultrasonic technique. Mater. Charact. 2011, 62, 275-286. [CrossRef]

22. Matlack, K.H.; Bradley, H.A.; Thiele, S.; Kim, J.Y.; Wall, J.J.; Jung, H.J.; Qu, J.; Jacobs, L.J. Non-linear ultrasonic characterization of precipitationin 17-4PH stainless steel. NDT E Int. 2015, 71, 8-15. [CrossRef]

23. Pruell, C.; Kim, J.Y.; Qu, J.; Jacobs, L.J. Evaluation of fatigue damage using non-linear guided waves. Smart Mater. Struct. 2009, 18, 035003. [CrossRef]

24. Rauter, N.; Lammering, R. Impact Damage Detection in Composite Structures Considering Non-linear Lamb Wave Propagation. Mech. Adv. Mater. Struct. 2015, 22, 44-51. [CrossRef] 
25. Lissenden, C.J.; Liu, Y.; Rose, J.L. Use of non-linear ultrasonic guided waves for early damage detection. Guided Waves 2015, 57, 206-211. [CrossRef]

26. Xiang, Y.; Zhu, W.; Deng, M.; Xuan, F. Experimental and numerical studies of non-linear ultrasonic responses on plastic deformation in weld joints. Chin. Phys. B 2016, 25, 024303. [CrossRef]

27. Matlack, K.H.; Kim, J.Y.; Jacobs, L.J.; Qu, J. Experimental characterization of efficient second harmonic generation of Lamb wave modes in a non-linear elastic isotropic plate. J. Appl. Phys. 2011, 109, 014905. [CrossRef]

28. Jiao, J.; Meng, X.; He, C.; Wu, B. Non-linear Lamb wave-mixing technique for micro-crack detection in plates. NDT E Int. 2017, 85, 63-71.

29. Zhu, W.; Xiang, Y.; Liu, C.; Deng, M.; Xuan, F. A feasibility study on fatigue damage evaluation using non-linear Lamb waves with group-velocity mismatching. Ultrasonics 2018, 90, 18-22. [CrossRef]

30. Wan, X.; Tse, P.W.; Xu, G.H.; Tao, T.F.; Zhang, Q. Analytical and numerical studies of approximate phase velocity matching based non-linear S0 mode Lamb waves for the detection of evenly distributed microstructural changes. Smart Mater. Struct. 2016, 25, 045023. [CrossRef]

31. Aleshin, V.; Delrue, S.; Trifonov, A.; Matar, O.B.; Abeele, K.V.D. Two dimensional modeling of elastic wave propagation in solids containing cracks with rough surfaces and friction-Part I: Theoretical background. Ultrasonics 2018, 82, 11-18. [CrossRef] [PubMed]

32. Delrue, S.; Aleshin, V.; Truyaert, K.; Matar, O.B.; Abeele, K.V.D. Two dimensional modeling of elastic wave propagation in solids containing cracks with rough surfaces and friction-Part II: Numerical implementation. Ultrasonics 2018, 82, 19-30. [CrossRef] [PubMed]

33. Liu, Y.; Chillara, V.K.; Lissenden, C.J. On selection of primary modes for generation of strong internally resonant second harmonics in plate. J. Sound Vib. 2013, 332, 4517-4528. [CrossRef]

34. Deng, M.; Wang, P.; Lv, X. Experimental verification of cumulative growth effect of second harmonics of Lamb wave propagation in an elastic plate. Appl. Phys. Lett. 2005, 86, 124104. [CrossRef]

35. Pruell, C.; Kim, J.Y.; Qu, J.; Jacobs, L.J. A non-linear-guided wave technique for evaluating plasticity-driven material damage in a metal plate. NDT E Int. 2009, 42, 199-203. [CrossRef]

36. Liu, Y.; Kim, J.Y.; Jacobs, L.J.; Qu, J.; Li, Z. Experimental investigation of symmetry properties of second harmonic Lamb waves. J. Appl. Phys. 2012, 111, 053511. [CrossRef]

37. Li, W.; Cho, Y. Thermal Fatigue Damage Assessment in an Isotropic Pipe Using Non-linear Ultrasonic Guided Waves. Exp. Mech. 2014, 54, 1309-1318. [CrossRef]

38. Deng, M.; Wang, P.; Lv, X. Influences of interfacial properties on second-harmonic generation of Lamb waves propagating in layered planar structures. J. Phys. D Appl. Phys. 2006, 39, 3018-3025. [CrossRef]

39. Zhao, J.; Chillara, V.K.; Ren, B.; Cho, H.; Qiu, J.; Lissenden, C.J. Second harmonic generation in composites: Theoretical and numerical analyses. J. Appl. Phys. 2016, 119, 064902. [CrossRef]

40. Zhao, Y.; Li, F.; Cao, P.; Liu, Y.; Zhang, J.; Fu, S.; Zhang, J.; Hu, N. Generation mechanism of non-linear ultrasonic Lamb waves in thin plates with randomly distributed micro-cracks. Ultrasonics 2017, 79, 60-67. [CrossRef]

41. Liu, Y.; Lissenden, C.J.; Rose, J.L. Strongly cumulative second harmonic generation in a plate with quadratic non-linearity: Finite element simulation. AIP Conf. Proc. 2013, 1511, 151-158.

42. Zhu, W.; Deng, M.; Xiang, Y.; Xuan, F.; Liu, C. Second Harmonic Generation of Lamb Wave in Numerical Perspective. Chin. Phys. Lett. 2016, 33, 104301. [CrossRef]

43. Deng, M. Cumulative second-harmonic generation of Lamb-mode propagation in a solid plate. J. Appl. Phys. 1999, 85, 3051-3058. [CrossRef]

44. Xiang, Y.; Zhu, W.; Deng, M.; Xuan, F.; Liu, C. Generation of cumulative second-harmonic ultrasonic guided waves with group velocity mismatching: Numerical analysis and experimental validation. EPL 2016, 116, 34001. [CrossRef]

45. Zhao, Y.; Qiu, Y.; Jacobs, L.J.; Qu, J. Frequency-Dependent Tensile and Compressive Effective Moduli of Elastic Solids with Randomly Distributed Two-Dimensional Microcracks. J. Appl. Mech. 2015, 82, 081006. [CrossRef]

46. Solodov, I.Y.; Krohn, N.; Busse, G. CAN: An example of nonclassical acoustic nonlinearity in solids. Ultrasonics 2002, 40, 621-625. [CrossRef]

47. Meurer, T.; Qu, J.; Jacobs, L.J. Wave propagation in nonlinear and hysteretic media-A numerical study. Int. J. Solids Struct. 2002, 39, 5585-5614. [CrossRef] 
48. Zhao, Y.; Qiu, Y.; Jacobs, L.J.; Qu, J. A micromechanics model for the acoustic non-linearity parameter in solids with distributed microcracks. AIP Conf. Proc. 2016, 1706, 060001.

49. Müller, M.F.; Kim, J.Y.; Qu, J.; Jacobs, L.J. Characteristics of second harmonic generation of Lamb waves in nonlinear elastic plates. J. Acoust. Soc. Am. 2010, 127, 2141-2152. [CrossRef]

(C) 2020 by the authors. Licensee MDPI, Basel, Switzerland. This article is an open access article distributed under the terms and conditions of the Creative Commons Attribution (CC BY) license (http://creativecommons.org/licenses/by/4.0/). 